\title{
Notes sur diverses formules relatives à l'écoulement de l'eau dans les conduites et les aqueducs de grandes dimensions
}

par M. Hubie, Inginieur des Ponts et Chaussées.

\section{I. - Considérations générales}

Le problème de l'écoulement de l'eau dans les aquedues el les conduites forcées a été étudié par d’éminents hydrauliciens tant en France qu'à l'étranger, et il semble bien que tout ou à peu près ait été écrit sur le sujel, el que mis en présence d'un cas concret, l'ingénieur n'ait qu'à puiser, dans le vaste arsenal des formules de toutes sortes qui s'offre à lui, celle qui lui paraîtra la plus simple, la plus commode et la mieux adaptée au problème qui lui est posé. Ceci est vrai tant qu'il s'agit d'ouvrages de dimensions courantes, mais ne l'est plus dès que celles-ci sortent de la moyenne correspondant aux expériences ayant servi de base aux formules dont nous venons de parler. Or, ici le problème à résoudre est particulièrement grave, car une légère diminution des dimensions des grands aqueducs entraîne une économie très importante sur l'ensemble de l'ouvrage. D'autre part, si l'on ne considère que les conduites forcées, l'usage du béton armé pour la construction de celles-ci tend à se généraliser pour des diamètres et des charges de plus en plus considérables et les renseignements expérimentaux sur le sujet manquent presque totalement en France.

C'est ainsi que nous avons eu à étudier un aqueduc circulaire de $4 \mathrm{~m}$. 50 de diamètre, capable de porter, lorsque le niveau de l'eau atteint les $3 / 4$ de la hauteur, 1 million de $\mathrm{m}^{3}$ par jour avec une pente de 12 centimètres par kilomètre. Les siphons prévus sont composés de quatre tuyaux en béton armé de 2 mètres de diamètre. Leur flèche pourra atteindre 75 mètres et la perte de charge admise est de $40 \mathrm{~cm}$. par $\mathrm{km}$.

Ces ouvrages rentrent dans la catégorie de ceux de dimensions exceptionnelles que nous avons en vue : il n'existe pas en effet d'adduction comparable en France et mème en Europe. Seuls quelques égouts de grandes villes sont de grandeur approchante. Par contre, en Amérique, les ouvrages similaires ne manquent pas : ceux des eaux de New-York en particulier (Croton, Catskill, etc.).

Nous nous proposons dans la présente Note de comparer entre elles les formules les plus usuelles pour les gros débils, de faire connaître les résultats d'expériences el les formules nouvelles en usage en Amérique, enfin de donner un abaque basé sur ces dernières formules, et pouvant servir à la fois aux conduites et aux aqueducs.

\section{II. - Aqueducs a Écoulement libre. \\ Comparaison des formules usuelles.}

Formule de Bazin. - C'est la plus usuelle en France; elle est de la forme :

avec :

$$
\mathrm{U}=\mathrm{C} V \overline{\mathrm{R}} \overline{\mathrm{I}}
$$

$$
\mathrm{C}=\frac{87 \gamma \overline{\mathrm{R}}}{V \overline{\mathrm{R}}+\gamma}
$$

où $R$ est le rayon moyen en mètres, I la pente, $U$ la vitesse en mètres par seconde et $\gamma$ un coefficient variable avec la nature des parois. Le point délicat est de savoir quel coefficient il faut adopter. Dans les aqueducs de dérivation d'eau potable, la sur face intérieure est recouverte d'un enduit lisse en ciment pour lequel le $\gamma$ indiqué par Bazin est 0,06. Or, l'expérience a montré que cette hypothèse clait trop oplimiste el que le coefficient de rugosité est plutôl voisin de 0,16 donné pour les parois an maçonnerie de moellons. Celte valeur est préconiséc par M. Eydoux, dans son cours d'hydraulique professé à l'Ecole nationale des Ponts et Chaussées.

Les expériences failes sur les aquedues de la ville de Pạris de diamètre compris entre 2 mètres el $2 \mathrm{~m}$. 50, domnent $\gamma=0.12$.

Si nous appliquons celle valeur à l'aqueduc qui nous accupe, nous oblenons le résultal suivant :

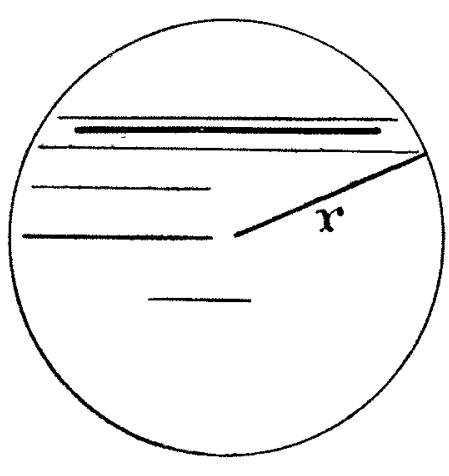

Fig. 1

Rayon $r$

Section mouillée

$$
\mathrm{S}=\frac{2 \pi r^{2}}{3}+\frac{r^{2} V \overline{3}}{4}=\frac{r^{2}}{12}(8 \pi+3 V \overline{3})=2,52 r^{2}
$$

Périmètre mouillé

$$
x=\frac{4 \pi r}{3}
$$

Rayon moyen

$$
\mathrm{R}=\frac{\mathrm{S}}{\%}=\frac{r}{16 \pi}(8 \pi+3 \mathrm{~V}, 3)=0,6 r
$$

Pour

$$
\text { 2 } r=4 \mathrm{~m} .50 \text { on a } \mathrm{R}=1 \mathrm{~m} .35 \mathrm{~V} \overline{\mathrm{R}}: 1,16
$$$$
\mathrm{S}=12 \mathrm{~m}^{2} 76
$$

$$
\begin{gathered}
\mathrm{U}=\frac{87 \times 1,16}{1,16+0,12} \times V \overline{\mathrm{RI}}=79 \vee \overline{\mathrm{R} 1}=79 \times 0,01279 \ldots \\
\ldots=1 \mathrm{~m} .01
\end{gathered}
$$

Formule de Manninf. - C'est la formule:

$$
\mathrm{U}=\mathrm{C} \mathrm{R}^{\frac{2}{3}} / \mathrm{T}
$$

avec $\mathrm{C}=77$ pour les parois unies. 
Elle donne ici :

$$
\mathrm{U}=77(1,35)^{\frac{2}{3}} 0,011=1 \mathrm{~m} .035
$$

Formule de Ganguillet et Kutter. - Cetle formule a été très utilisée à l'étranger, mais sa complication lui en fait préférer d'autres : celle de Bazin, ou celles dont nous parlerons plus bin.

C'est lit formule

$$
\mathrm{U}=\mathrm{C} V \overline{\mathrm{R} 1}
$$

avec

$$
\mathrm{C}=\frac{23+\frac{0,00155}{\mathrm{l}}+\frac{1}{\mathrm{~N}}}{1+\left(23+\frac{0,00155}{\mathrm{I}}\right) \frac{\mathrm{N}}{V \mathrm{R}}}
$$

où $\mathrm{N}$ joue le ròle de $\gamma$ dans la formule de Bazin.

Là encore une diffieulté réside dans le choix de N.

(n a $\mathrm{N}=0,01$ pour les parois en ciment.

$\mathrm{N}=0.013$ pour les parois en moellons et briques.

Par analogie avee la formule de Bazin, il est à prévoir que la première valeur est trop optimiste. En effet :

$$
\begin{aligned}
& \mathrm{N}=0.01 \text { donne } \mathrm{C}=104 \text { et } \mathrm{U}=1 \mathrm{~m} .32 \\
& \mathrm{~N}=0.013 \text { donne } \mathrm{C}=80,5 \text { et } \mathrm{U}=1 \mathrm{~m} .03
\end{aligned}
$$

résultat équivalent aux deux précédents.

L'exemple choisi montre que les formules passées en revue sont sensiblement équivalentes entre elles; même pour des dimensions d'aqueducs s'écartant considérablement de celles à l'aide desquelles elles furent établies. Ce premier résultat est déjà très intéressant et l'on peut en déduire qu'il est pratiquement inutile, tout au moins dans la limite d'application sûre de ces formules, de s'embarrasser d'un grand nombre d'entre elles. En France, la formule de Bazin est généralement employée.. Cetle première discussion nous montre qu'elle répond parfaitement aux besoins de la pratique courante, eu égard à la précision qu'on peut espérer en ces sortes de questions et qu'il n'y a pas à chercher à lui en substituer une autre. La valeur de $\%$ est de l'ordre de 0.12 à 0.16 pour une paroi recouverte d'un enduit lissé au ciment.

La question qui se pose alors est de savoir si les résultats que la formule de Bazin fournit pour des ouvrages de dimensions exceptionnelles sont conformes à la réalité et si l'extrapolation que l'on fait dans ce cas n'est pas trop hardie. C'est que ce nous essaierons de déterminer dans un instant $(\$ \mathrm{~V})$.

\section{III. - Conduites forcées en béton armé.}

\section{Comparaison des formules usuelles.}

A. Formules particulières aux conduites. - La seule indication précise que nous ayons recueillie à propos de ce problème, se trouve dans le cours de M. Eydoux où il est proposé d'uliliser la formule de Maurice Lévy pour conduites métalliques en majorant le coefficient.

Formule de Maurice Lévy. - Elle s'exprime :

$$
\mathrm{U}=25 \sqrt{\frac{\overline{\overline{\mathrm{D} J}}}{2}\left(1+3 \sqrt{\left.\frac{\overline{\mathrm{D}}}{2}\right)}\right.}
$$

où $\mathrm{D}$ est le diamètre en mètres et $\mathrm{J}$ la perte de charges.

Pour les conduites métalliques de grand diamètre, le coefficient n'est que de 20.5 , ce qui représente une majoration de 1.22 pour le béton armé.
Appliquons ces données aux siphons prévus à l'étude précitée :

$$
\mathrm{D}=2 \text { mètres } \quad \text { et } \quad \mathrm{J}=0.0004
$$

On obtient :

$$
\mathrm{C}=25 V \overline{0,000 /(1+3)}=1 \text { mètre }
$$

Formule de Flamant. - D'après ce que nous venons de voir, et à défaut d'autre indicalion, il semble que les formules pour conduites moyennement incrustées qui sont optimistes pour le métal puissent convenir au béton armé. La formule à appliquer est alors :

$$
J=0,00090 \frac{U^{\frac{7}{4}}}{D^{\frac{5}{4}}}
$$

Elle donne dans l'exemple choisi : $\mathrm{U}=1 \mathrm{~m} .02$.

Formule d'Unwin. - La formule pour fonte neuve semble convenir ici :

$$
\mathrm{J}=0,00084 \frac{\mathrm{U}^{2}}{\mathrm{D}^{1.25}}
$$

Elle donne: $\mathrm{U}=1 \mathrm{~m} .0 .6$ dans le cas étudié.

Formule de Mougnié. - On sait que MI. Mougnié a entrepris une étude critique des résultats d'Unwin et est arrivé à la formule suivante :

$$
J=0,002 \frac{\mathrm{U}^{2}}{\mathrm{D}^{1: 25}}
$$

applicable à la fonte en service courant.

On obtient $U=0.70$ ce qui est franchement faible. En corrigeant ce nombre d'après l'indication de M. Eydoux, on obtient : $\mathrm{U}=0.70 \times 1.22=0.854$, ce qui doit être encore faible.

Formule de Geslain. - M. Geslain, ingénieur au Service municipal de la Ville de Paris, a proposé, à la suite d'études sur les conduites existantes, la formule suivante :

$$
\mathrm{U}=(0,96+0,24 n) \mathrm{D}^{0,75-0,10 n} \mathrm{~J}^{050+0,0.5 n}
$$

avec

$$
\begin{aligned}
& n=1-\text { parois lisses } \\
& n=\frac{2}{3} \text { - fonte neuve } \\
& n=\frac{1}{3} \text { - fonte légèrement incrustée } \\
& n=0 \text { - fonte incrustée en service courant. }
\end{aligned}
$$

On obtient dans le cas présent avec :

$$
\begin{aligned}
& n=0-\mathrm{U}=1 \mathrm{~m} .01 \\
& n=\frac{1}{3}-\mathrm{U}=1 \mathrm{~m} .06 \\
& n=\frac{2}{3}-\mathrm{U}=1 \mathrm{~m} .09 .
\end{aligned}
$$

D'après les considérations précédentes, il conviendrait d'adopter, pour le béton armé,

$$
n=\frac{1}{3} \quad \text { et } \quad \mathrm{U}=1 \mathrm{~m} .06 \text {. }
$$

B. Formules communes aux conduites ct aux aqueducs. Certains auteurs préconisent l'emploi des mèmes formules dans les deux cas, en faisant sculement varier les coefficients. 
Formule de Bazin. - M. Eydoux, dans son cours d'hydraulique, propose demployer la formule de Bazin avec le coefficient des parois lisses $r=0.06$.

$$
\mathrm{U}=\frac{\delta 7 \mathrm{R} V \mathrm{~J}}{i+V \overline{\mathrm{R}}}
$$

où on a $R=\frac{D}{4}$ et $J$ est la perte de charge au lieu de la pente superficielle.

Elle donne dans le cas actuel :

$$
\mathrm{U}=\frac{8 \mathrm{i} \times 0, \overline{\mathrm{c}} \times 0,19}{0,06+V \overline{0.5}}=1 \mathrm{~m} .14
$$

résultat paraissant trop optimiste.

Formule de Ganguillet et liutter. - Comme pour la formule de Bazin, $\mathrm{R}=\frac{\mathrm{D}}{4}$ et la perte de charge $\mathrm{J}$ remplace la pente $\mathrm{I}$.

$\mathrm{N}$ varie de 0.010 à 0.019 avec

$\mathrm{N}=0.014$ pour les conduites en acier rivé neuves

$\mathrm{N}=0,013$ pour les conduites en fonte neuve.

Ici, l'application de $\mathrm{N}=0.013$ donne $\mathrm{U}=0.87$.

Si l'on compare entre eux les résultats précédents on est frappé de leur discordance, beaucoup plus sensible ici que dans le cas des aqueducs à écoulement libre : les résultats pour un mème exemple varient de $0 \mathrm{~m}$. 85 (et même $0 \mathrm{~m}$. 70) à $1 \mathrm{~m}$. 14 avec une moyenne de l'ordre de 1 mètre à $1 \mathrm{~m}$. 05 . Il est bien difficile d'en tirer une conclusion ferme. On peut toutefois remarquer que si l'on ne s'occupe que des résultats les plus concordants : formules de Maurice Lévy, de Flamant, d'Unwin, de Geslain, on paraît obtenir des vitesses admissibles en première approximation en faisant usage pour le béton des coefficients établis pour la fonte légèrement incrustée (c'est le coefficient 25 de la formule de Maurice Lévy qui milite en faveur de cette manière de voir).

L'incertitude qui subsiste malgré tout sur la formule à employer ne peut évidemment être levée que par l'expérience. Or, celle-ci a fait jusqu'à présent presque complètement défaut en France. On commence seulement à en entreprendre dans les usines hydrauliques comportant des conduites forcées en béton armé. Nous avons donc été amené, pour lever l'incertitude en question, à nous documenter sur les expériences qui ont été faites à l'étranger, particulièrement en Amérique, sur des conduites en béton armé de gros diamètre. Nous en avons profité pour noter également les résultats expérimentaux obtenus aux Etats-Unis sur l'écoulement de l'eau dans les aqueducs en conduite libre de grandes dimensions. Nous exposerons successivement ces deux questions.

\section{IV. - EXPÉriences et formules aMÉricaines pour les CONDUITES EN BÉTON $\left({ }^{1}\right)$.}

Les renseignements suivants sont extraits d'une brochure du colonel Longley de la "Loch joint Pipe Cie".

Lorsque les conduites en bélon armé sont faites avec soin, les joints ne sont pas perceptibles intérieurement et la paroi interne est très lisse. On arrive à ce résultal en employant un dosage riche, des cailloux bien échantillonnés et des moules mélalliques huilés.

Dans ces conditions, les qualités hydrauliques d'une conduile sont excellentes, tout à fait comparables à celles d'une conduite

${ }^{(1)}$ Nous devons exprimer toute notre reconnaissance à $M$. Imbeaux, ingénieur en Chef des Ponts et Chaussées, qui s'est très aimablement entremis pour nous procurer la documentation nécessaire auprès de ses amis, le grand hydraulicien américain Allen Hazen et le Colonel Longley, ancien chef du Service du Water Supply de l'Armée américaine en France. mélallique el se conservent à peu près intactes pendant un grand nombre d'amées. C'est là le fail d'expérience le plus intéressant : handis que la capacilé d'écoulement d'une conduile métallique. diminue rapidement d'abord, puis plus lentement, - par suite de la formation de dépòts el de corrosions, - celle d'une conduite en béton se conserve à peu de chose près sans changements. Pour le calcul du débil des conduites, nous avons noté deux formules appartenant à la catégoric des formules monômes $\mathrm{U}=\mathrm{Cl})^{\mathrm{k}} \mathrm{J}^{\mathrm{y}}$, la formule de Scobey et celle de Williams-I Iazen.

Formule de Scobey. - Elle s'exprime par la relation :

$$
\mathrm{U}=3 / 4 \mathrm{D}^{0,625} \mathrm{~J}^{0,5}
$$

oul

$$
\mathrm{J}=0,00086^{\circ} \frac{\mathrm{U}^{2}}{\mathrm{D}^{1.25}}
$$

On voit qu'elle est identique ou presque (0.00086 au lieu de 0.00084 ) à celle de Unwin pour la fonte neuve, ce qui est une première confirmation de ce que nous avons annoncé précédemment. Si on l'applique à l'exemple déjà choisi, on trouve :

$$
\mathrm{U}=1 \mathrm{~m} \text {. } 08
$$

Nous dommons un abaque établi d'après la formule de Scobey pour tuyaux en béton (Annexe III).

Formule de IIlliams-Hazen. - Elle est de la forme

$$
\mathrm{U}=0,8, \mathrm{C} \mathrm{r}^{0,60} \mathrm{~J}^{0,51}
$$

où $r$ est le rayon moyen $\left(\frac{D}{4}\right)$ et C un coefficient variable suivant la nature du tuyau el la vilesse. (Nous avons conservé à la formule sa forme américaine, comportant un coefficient numérique devant la constante $C$. Cela permet en outre de se servir du coefficient $\mathrm{C}$ tel qu'on le trouve dans les ouvrages américains.)

La valeur moyenne de $\mathrm{C}$ est de :
90 pour l'acier rivé.
100 pour la fonte.
128 pour le béton armé.

L'augmentation de débit des conduites en béton par rapporl à celles en fonte serail donc de 1,28 au lieu de 1,22.

D'autre part, C varie avec le diamètre el la vilesse pour une même nature de parois. Voici les valeurs de $\mathrm{C}$ pour le béton armé.

\begin{tabular}{|c|c|c|c|c|c|c|c|}
\hline Vitesses & $0 \mathrm{~m} 30$ & $1 \mathrm{~m} .50$ & $1 \mathrm{~m}$. & $1 \mathrm{~m} .5$ & $2 \mathrm{~m}$. & $2 \mathrm{~m} .50$ & $3 \mathrm{~m}$. \\
\hline Diamètres & & & & & & & \\
$0 \mathrm{~m} .30$ & 134 & 131 & 122 & 117 & 114 & 112 & 111 \\
$0 \mathrm{~m} .50 \mathrm{t}$ & 13. & 132 & 124 & 11.1 & 116 & 114 & 113 \\
$1 \mathrm{~m}$. & 138 & 135 & 127 & 123 & 120 & 118 & 116 \\
$1 \mathrm{~m} .510$ & 140 & 138 & 129 & 125 & 122 & 120 & 118 \\
$3 \mathrm{~m}$. & 143 & 141 & 130 & 126 & 123 & 121 & 119 \\
$2 \mathrm{~m} .500$ & 146 & 143 & 132 & 128 & 125 & 123 & 121 \\
$3 \mathrm{~m}$. & 149 & 146 & 134 & 130 & 127 & 125 & 123 \\
$3 \mathrm{~m} .500$ & 152 & 149 & 136 & 131 & 118 & 126 & 12. \\
\hline
\end{tabular}

Pour faire usage de la formule, il faut d'abord partir de la valeur moyenne, soit 128; on en déduit la vilesse approchéc, puis la valeur exacte de $\mathrm{C}$ et enlin la vitesse exacte.

Dans le cas qui nous sert d'exemple, nous avons :

$$
\mathrm{D}=2 \mathrm{~m} . \quad \mathrm{U}=1 \mathrm{~m} . \quad \text { d'où } \quad \mathrm{G}=130
$$

ce qui donne :

$$
\mathrm{U}=1 \mathrm{~m} .06
$$

(1) En unités anglaises, $U=1,31825 \mathrm{C}_{1} \mathrm{r}^{0,63} \mathrm{~J}^{0.54}$. 
L'arlicle du colonel Longley donne le résultat d'expériences lailes par Scobey sur des conduites en béton armé.

Des essais malheureusement trop peu nombreux ont porté sur la même conduite à intervalle de 2 à 4 ans; ils ont montré que $\mathrm{C}$ ne diminuait pas (sauf dants le cas de dépôt de vase dans le (uyau); d'autres ont consisté à déterminer $\mathrm{C}$ pour 44 conduites de diamètres variant de $0 \mathrm{~m}$. 300 à $5 \mathrm{~m} .25$ dont plusieurs de plus de ' 2 mètres. L'allure générale des résultats est la suivante. Pour les conduites faites avec soin, à parois lisses et à joints soignés, C est très élevé et varie de 135 à 155 maximum. Pour les conduites ne présentant rien d'anormal ni en soins, ni en mallaçons, C varie de 100 à 135. A chaque fois que C est plus petit que 100 , une raison particulière existe : conduite encrassée, joints grossiers, surface interne rugueuse, chef de chantier inexpérimenté, ele. Le minimum est de 86. Nous donnons le détail des expériences de Scobey (annexe I) car. c'est là, à notre connaissance le premier résultal expérimental d'en semble, concernant les grands diamètres et le béton armé.

Pour ce qui est du choix à faire entre telle ou telle formule, nous voyons que la formule de Williams-Hazen donne des résulLats comparables à celle de Scobey (ou ce qui est la même chose, celle d'Unwin pour la fonte neuve) et à celle de Geslain pour la fonte neuve ou légèrement incrustée. Les vitesses dans l'exemple choisi varient de $1 \mathrm{~m} .06$ à $1 \mathrm{~m} .09$.

Nous pouvons en tirer une première conclusion, à savoir que l'hypothêse que nous avons faite en première approximation à la fin du paragraphe précédent est légitime et que l'on obtient de bons résultats pour le béton armé en se servant des coefficients donnés pour la fonle neuve ou légèrement incrustée.

D'autre part, les expériences américaines ont montré que le coefficient d'écoulement $\mathrm{C}$, ou son équivalent dans les autres formules, reste sensiblement constant pour le béton armé, tandis que, dans le cas de la fonte, il diminue d'abord rapidement, puis plus lentemenl, à la suite des incrustations. En conséquence, il n'y a pas à faire intervenir le facteur temps pour le calcul des luyaux de la première espèce.

En résumé, nous conclurons de cetle partie de notre étude que l'on peut appliquer les formules d'Unwin ou de Geslain pour la fonte légèrement incrustée au cas du béton armé sans se préoccuper d'une diminution de la capacité d'écoulement avec le temps. La deuxième formule qui est française el est en usage au Service municipal de la Ville de Paris nous semble particulièrement intéressante.

\section{V. - EXPÉriences ET ForMULES MMÉricAiNes pour LES GRANDS AQUEDUCS.}

En Amérique, comme en Europe, les expériences sur les aqueducs de grandes dimensions sont rares.

Des mesures directes de vitesse ont été faites sur certains d'entre eux. Nous avons relevé les résultats suivants. Pour l'aqueduc de Sudbury (voir fig. 2) la vitesse moyenne s'exprime bien par la formule :

$\mathrm{U}=127 r^{0,62} 1^{0,50}$ (unités anglaises) alors que la formule de Scobey donnait avec ces unités :

$$
\mathrm{U}=128 r^{0,625} 1^{0,50}
$$

La concordance est presque parfaile.

Pour l'aquedue de New-Croton, on a trouvé :

$$
\mathrm{U}=124 r^{0,56} 1^{0,50}
$$

Formule de Williams-Hazen. - Pratiquement cette formule peut convenir aux conduiles et l'on arrive à celte conception de se servir toujours de la même formule dans tous les cas, comme cela avait été déjà tenté, - avec moins de succès il est vrai, - pour les formules de Bazin et de Ganguillet et Kutter.
Un certain nombre de valeurs de $\mathrm{C}$ ont été déterminées pour de grands aqueducs. Nous en donnons plus loin un extrait qui montre que la valeur moyenne trouvée est :

$$
\mathrm{C}=127 \text { (valeurs extrêmes } 114 \text { et 135). }
$$

Il est remarquable de vérifier encore une fois l'identilé de cette valeur avec celle relative aux conduites en béton. Appli-

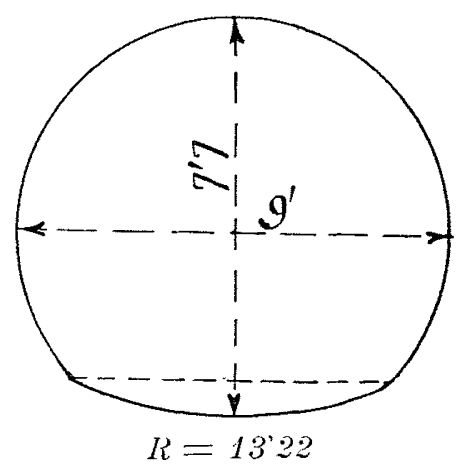

Fig. 2

quée à l'aqueduc envisagé précédemment, on trouve avec cette formule :

$$
\mathrm{C}=127 \quad \mathrm{U}=1 \mathrm{~m} .01
$$

C'est la vitesse trouvée précédemment par l'emploi de la formule de Bazin. Nous avons vérifié sur d'autres exemples que cette concordance se maintient remarquablement. Nous pouvons dès lors répondre à la question posée à la fin du $\$ 2$ par l'affirmative. L'extrapolation de la formule de Bazin (et des formules analogues) est légitime pour les grands diamètres et nous avons maintenant des résultats d'expériences pour asseoir notre opinion. Voici d'ailleurs le résumé de quelques mesures de $\mathrm{C}$ faites sur des aqueducs américains.

Aqueduc des Catskill. - $\mathrm{i}=0.0001156$ - Débit $\mathrm{Q}=22 \mathrm{~m}^{3} \mathrm{sec}$

Aqueduc couvert et en tranchée; moyenne de 10 expériences 133

Tunnel en charge et en tranchée; moyenne de 17 expériences 136

Nouvel aqueduc du Croton. $-\mathrm{i}=0.000187 \quad \mathrm{Q}=13 \mathrm{~m}^{3} 1$

Premières expériences....................... 125

Nouvelles expériences vers l'amont . . . . . . . . . . . 102

Nouvelles expériences vers l'aval................ 123

Ancien aqueduc de Croton. $-\mathrm{i}=0.000217$

Première expérience.................... 121

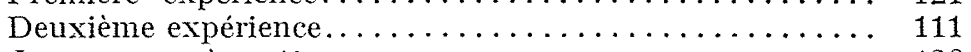

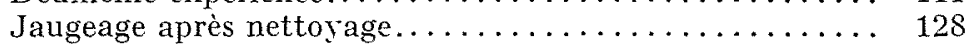

Adopté par mesure de sécurité................ 118

Wachusett aqueduc.

Neuf........................... 137

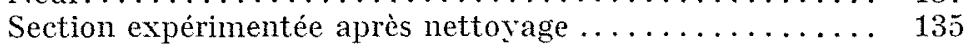

Aqueduc de Subdury.

Première expérience. .................... 135

Section préparée spécialement.............. 112

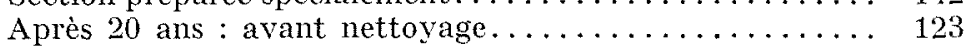

Après 20 ans : après nettoyage............... 137

Détroit.

A 21 ans : briques sans enduit.............. 115

Aqueduc de Saint-Louis.

Après un certain temps, incrustations calcaires 3 cent. par place......................... 114

Tunnel de Chicago $(\mathrm{D}=2 \mathrm{~m} .40) \ldots \ldots \ldots \ldots \ldots \ldots \ldots . \ldots \ldots . .114$

Manchester. Aqueduc de Thirlmere. -- $\mathrm{i}=0.00379 \quad \mathrm{Q}=2 \mathrm{~m}^{3} 650$

Diamètre 2 m. $15 \ldots \ldots \ldots \ldots \ldots \ldots \ldots \ldots \ldots \ldots \ldots \ldots \ldots \ldots \ldots$

Glascou. Aqueduc lac Katrine. - $\mathrm{i}=0.00015 \mathrm{Q}=3 \mathrm{~m}^{3} 700 \ldots \quad 131$

Nous venons de voir qu'en Amérique on cherche à se servir de la mème formule pour les aqueducs et les conduites. Voici 
à tilre d’indication, les limiles entre lescuucles varic le coefficient

C dans différents cas :

Conduites en fonte neuve....... 111 a 116

Conduites en fonte usagée....... 16 à 112 (moyenne 100$)$

Conduites acier rivé neuf......... 97 à 142

Conduites acier rivé usaoé .........

Conduites beton armé......... 12s (movemne)

Conduites en bois............ 113 a 129

Aquedues en maconnerie.......... 114 a 1.5

Egouts en briques............ 10.2 à 111

Aquedues en planches. . . . . . . 113 à 120

Camal lisse................ 136 à 145

Camal en terre............. 15 a 61

Canal à parois très rugueuses..... 30 a

\section{VI. - CONCLLSIONS}

De loute celle élude des expériences ot formules américaines relatives aux aqueducs et conduites en bélon armé de grandes dimensions, nous pouvons tirer les conclusions suirantes:

$1^{\circ}$ Concernant les aqueducs avec enduit lisse au ciment, la formule de Bazin (avec $\gamma$ voisin de 0.12 à 0.16 ) donne de bons résultats mème pour les grands diamètres et l'on ne voil pas de raison de s'en écarter en France où elle est d'un emploi général; elle suffit à elle seule à résoudre les problèmes posés, avec une approximation suffisante en pratique.

$2^{\circ}$ Concernant les conduites en béton armé, la formule de Geslain, consacrée par un grand nombre d'expériences sur des conduites de diamètre important (jusqu'à $1 \mathrm{~m}$. 25) en fonle ou en acier, peut ètre appliquée au béton armé à condition de faire usage du coefficient $n=\frac{1}{3}$ applicable à la fonte légèrement incrustée.

$3^{\circ}$ Concernant les formules américaines, celle de WilliamsHazen présente des particularités qui la rendent utile à connaître.

a) Elle est de la forme monôme, ce qui la rend immédiatement calculable par logarithmes.

b) Elle semble aujourd'hui très répandue en Amérique oì elle tend à remplacer l'ancienne formule compliquée el incommode de Ganguillet et Kutter. Il est done intéressant pour suivre les expériences faites dans ce pays d'en connaître la signification.

c) Elle condense en une seule formule les résultats relalifs aux aqueducs et aux conduites, ce qui présente une commodité indéniable en ramenant tous les résultats d'expérience à la connaissance d'un seul coefficient $C$.

Pour toutes ces raisons, il nous a paru utile de construire un abaque pour faciliter son emploi et permettre les comparaisons avec les formules françaises mentionnées plus haut et l'interprétation des faits expérimentaux $\left(^{1}\right)$.

Nous devons observer en terminant que la présence de quatre variables $r, j, \mathrm{C}$ et $\mathrm{U}$ conduit à un nomogramme à double alignement comportant une charnière, mais dont la réalisation ne présente aucune particularité remarquable.

Enfin, il serait intéressant de contrôler les conclusions précédentes à l'aide d'expériences nouvelles.

\section{ANNEXE I}

\begin{tabular}{|c|c|c|c|}
\hline $\begin{array}{l}\text { Diamètre } \\
\text { intérieur }\end{array}$ & $\mid \begin{array}{c}\text { Longueur } \\
\text { de la } \\
\text { conduite }\end{array}$ & $\begin{array}{c}\text { G } \\
\text { Williams } \\
\text { Hazen }\end{array}$ & $\begin{array}{l}\text { Remarques de - cobey } \\
\text { relatives a la rugosite des parois. } \\
\text { des joints et a la construction }\end{array}$ \\
\hline $\mathrm{m}$ & $\mathrm{m}$ & 155,1 & $\begin{array}{l}\text { Moulé dans des formes en acier nettoyées à } \\
\text { l'huile à chaque opération. -- Surface inté- } \\
\text { rieure particulièrement lisse. - Joints } \\
\text { aussi lisses que le reste de la conduite. }\end{array}$ \\
\hline
\end{tabular}

(1) Le lecteur trouvera cet abaque dans les Annales des Ponts et Chaussées, $\mathrm{N}^{\mathrm{os}}$ janvier-février.
ANNEXE I (suile)

\begin{tabular}{|c|c|c|c|}
\hline $\begin{array}{l}\text { Diamitre } \\
\text { interieur }\end{array}$ & $\begin{array}{l}\text { 1.ongneur } \\
\text { die liz } \\
\text { rontluite }\end{array}$ & $\begin{array}{c}c \\
\text { Wiilitums } \\
\text { llazen }\end{array}$ & $\begin{array}{l}\text { Jemarues de seoboy } \\
\text { refalives a la rugosite des parois, } \\
\text { des joints et a lat construction }\end{array}$ \\
\hline 5,25 & 1.970 & 152,8 & $\begin{array}{l}\text { I.a surface de la conduite est particulièreemul } \\
\text { lisse el mème, un grand soin a été pris de } \\
\text { mettre en exuve le béton après avoir huile } \\
\text { les moules d'acier. }\end{array}$ \\
\hline 1,15 & 3.000 & 151,5 & $\begin{array}{l}\text { Les éléments étaient moulés dans des formes } \\
\text { en acier. - Conduite en alignement droit. }\end{array}$ \\
\hline 1,60 & 350 & 146.8 & $\begin{array}{l}\text { Moules intérieurs en acier. Interieur tres } \\
\text { lisse et propre après huit ans de service. }\end{array}$ \\
\hline 0.800 & 10 & 116,5 & Joints parfaits. \\
\hline 0.770 & $\ldots$. & 1.11 & $\begin{array}{l}\text { Tuyaux de } 1 \mathrm{~m} \text {. } 20 \text { moulés dans des formes } \\
\text { en bois recouvertes de feuilles al'acier. }\end{array}$ \\
\hline 2.800 & $\ldots$ & $1 \mid 2,7$ & \\
\hline 1.07 & 110 & 141.7 & $\begin{array}{l}\text { Moulé dans des formes en acier netloyées à } \\
\text { lhuile à chaque opération. -... Surface } \\
\text { intérieure particulièrement lisse. Joints } \\
\text { aussi lisses que le reste de la conduite. }\end{array}$ \\
\hline 1,15 & 3.000 & 138,3 & $\begin{array}{l}\text { Les éléments étaient moulés dans des formes } \\
\text { en acier. - Conduite en alignement droit. }\end{array}$ \\
\hline 4.10 & 2.560 & 136,8 & $\begin{array}{l}\text { Paroi interne moulée contre des formes } \\
\text { dlacier propres et huilées. J } \text { Béton soi- } \\
\text { gneusement lamé contre le moule pour } \\
\text { assurer une surface interne dense et lisse. -- } \\
\text { Surface quelque peu souillée. }\end{array}$ \\
\hline 0,920 & 2.210 & 136 & $\begin{array}{l}\text { Tuyaux de } 1 \mathrm{~m} \text {. } 80 \text { de longueur moulés sur le } \\
\text { sol dans des formes dacier. -.. Joints cal- } \\
\text { fatés avec grand soin. - Mortier à } 1 \text { de } \\
\text { ciment pour } 2 \text { de sable. }\end{array}$ \\
\hline 2,20 & 1.275 & 135.6 & \\
\hline 1,37 & 660 & 129,4 & \\
\hline 0,600 & 320 & 127.3 & $\begin{array}{l}\text { Tuyaux construits dans des moules en bois } \\
\text { tant internes qu'externes. - Des débris de } \\
\text { béton sont visibles à l'intérieur. }\end{array}$ \\
\hline $\begin{array}{l}0,77 \\
0,51\end{array}$ & $\overline{660}$ & $\begin{array}{l}126 \\
125\end{array}$ & $\begin{array}{l}\text { Eléments de } 0.90 \text { de longueur lavés avec } \\
\text { un lait de ciment avant mise en place. } \\
\text { Joints lisses. Exemple typique de ce } \\
\text { que donne une bonne pratique (1911). }\end{array}$ \\
\hline
\end{tabular}

123,3 Joints imparfaits. - Moules en acier huilés donnant une surface interne lisse.

$1.530122,8$ Elẹments de $1 \mathrm{~m} .20$ de longueur. - Moules en bois recouverts de feuilles d'acier.

Le coefficient $\mathrm{Cs}=0,33$ se rapporte plutôt à la surface envasée qu'à la nature mème de la paroi.

94,2 Eléments de $0 \mathrm{~m}$. 60, joints très grossiers. 93,1 Eléments de $0 \mathrm{~m}$. 90.

93 Pas de dépôts. 91,1 Pas de dépôts.

90,7 Eléments de $0 \mathrm{~m}$. 60.

90,2 Ouvrage de quelqu'un d'inexpérimenté. Dépôts dans la conduite.

88,6 Eléments de $0 \mathrm{~m}$. 60 .

87,5 Joints mauvais. - Surface interne rugueuse. 86,2 Joints grossiers, ouvrage de quelqu'un d'expérimenté. 